\title{
Event-Based State Estimation for a Class of Complex Networks with Time-Varying Delays: A Comparison Principle Approach
}

\author{
Wenbing Zhang ${ }^{a}$, Zidong Wang ${ }^{b, *}$, Yurong Liu ${ }^{a, d}$, Derui Ding ${ }^{c}$ and Fuad E. Alsaadi ${ }^{d}$
}

\begin{abstract}
The paper is concerned with the state estimation problem for a class of time-delayed complex networks with event-triggering communication protocol. A novel event generator function, which is dependent not only on the measurement output but also on a predefined positive constant, is proposed with hope to reduce the communication burden. A new concept of exponentially ultimate boundedness is provided to quantify the estimation performance. By means of the comparison principle, some sufficient conditions are obtained to guarantee that the estimation error is exponentially ultimately bounded, and then the estimator gains are obtained in terms of the solution of certain matrix inequalities. Furthermore, a rigorous proof is proposed to show that the designed triggering condition is free of the Zeno behavior. Finally, a numerical example is given to illustrate the effectiveness of the proposed event-based estimator.
\end{abstract}

\section{Index Terms}

Event-triggering; State estimation; Complex networks; Comparison principle; Time-varying delays.

\section{INTRODUCTION}

Nowadays, it is widely recognized that complex networks are capable of modeling various natural phenomena owing to their potential applications in the areas of image processing, neuronal synchronization and secure communication [36]. With the development of information technology, complex networks are ubiquitous, such as neural networks, ecosystems, social network, the Internet, the WWW and electrical power grids, etc. Since the seminal work on the "small-world" and "scale-free" properties in [1] and [38], complex networks have become an ongoing research topic that attracts ever-increasing attention in recent years. Up to now, there has been a rich body of results on the dynamics analysis issues of complex networks such as stability and stabilization [11], synchronization [27], [28], [31] and pinning control [2], [24].

As with the traditional dynamical systems, time-delays are ubiquitous in the implementation of practical complex networks because of the finite speed of transmission and spreading as well as congestions in signal transition [16],

This work was supported in part the Royal Society of the UK, the National Natural Science Foundation of China under Grants 61329301, 61374010, 61503328 and 61573246, and the Alexander von Humboldt Foundation of Germany.

${ }^{a}$ Department of Mathematics, Yangzhou University, Yangzhou 225002, China.

${ }^{b}$ Department of Computer Science, Brunel University London, Uxbridge, Middlesex, UB8 3PH, United Kingdom.

${ }^{c}$ Shanghai Key Lab of Modern Optical System, Department of Control Science and Engineering, University of Shanghai for Science and Technology, Shanghai 200093, China.

${ }^{d}$ Communication Systems and Networks (CSN) Research Group, Faculty of Engineering, King Abdulaziz University, Jeddah 21589, Saudi Arabia.

* Corresponding author. E-mail: Zidong.Wang@brunel.ac.uk 
[33], [44]. Since time-delays could lead to undesirable dynamic behavior such as oscillation and instability, it is vitally important to examine the impact from time-delays on dynamical performance [44]. As such, the last decade has witnessed a rapidly growing interest in the pinning control and synchronization problems for various kinds of complex networks with node delays [14], [41], coupling delays [11] or random sensor delays [4], [17]. For instance, the synchronization problem has been investigated in [26] for an array of linearly coupled identical connected neural networks by using variational method, where the coupling matrix is not restricted to be symmetric or irreducible. In [11], a delay-partitioning approach has been used to obtain a new delay-range-dependent criterion for the synchronization stability of complex dynamical networks.

It is worth noting that, in most works on the pinning control and the synchronization, it has been implicitly assumed that system states are fully accessible. This is, unfortunately, not always the case in practice [32]. For instance, only partial information about the network states is available in virus spreading networks and sensor networks. In multi-agent systems, the information of positions is usually utilized to achieve the consensus control, and the velocities are commonly discarded due to the expensive measurements. In this case, it is of both theoretical importance and practical significance to estimate the node states of complex networks by using the available measurements [25] so as to carry out subsequent tasks such as performance analysis and design. By now, some preliminary results have been reported on the state estimation problems of complex/neural/sensor networks [4], [21], [37]. For instance, the references [32] and [4] have provided estimator design approaches, in terms of linear matrix inequalities, for the systems with uncertain inner coupling and incomplete measurements as well as the systems with the random sensor delays, respectively. Nevertheless, the state estimation problem for more general complex networks with time-varying delays has not yet received adequate research attention, and this constitutes the first motivation for our current investigation.

In the execution of estimation algorithms, the sampled-data estimation strategy has been generally applied due to the increasing popularity of communication networks. In such a strategy, the sampling period is predetermined which might lead to over-provisioning of the real-time system hardware. In addition, the limited communication resources could also be overly consumed especially for the large-scale distributed systems including complex networks as a special case. Very recently, a novel communication protocol, namely, event-triggering mechanism, has been developed to overcome the aforementioned drawbacks, see [29], [34], [35], [39], [43], [45] and the reference therein. In comparison of the time-triggering case (i.e. the periodic sampling case), the input to the controller or estimator is updated only when a certain triggering condition is violated. Up to now, only limited work has been available in the literature on the synchronization of complex networks with event-triggering. For instance, a new distributed event-triggered mechanism has been introduced in [12] for pinning control synchronization of complex networks. The event-triggered distributed state estimation problem over sensor networks has been investigated in [15] for a class of discrete-time nonlinear stochastic systems with mixed network-induced phenomena.

When both the time-varying delays and event-triggering communication protocol are taken into simultaneous consideration, the estimator design problem for complex networks exhibits a few technical challenges. On one hand, it is well known that, due to the added delay-related terms, the frequently used Lyapunov-Krasovskii functional approach would inevitably suffer from increased complexity of the design schemes especially for large-scale systems. As such, the first difficulty would be how to develop a new method to reduce the effect from time-delays on the estimator design. Thus, the motivation of this paper is to derive a comparison principle to study the state estimation problem of time-delayed complex networks with event-triggering communication protocol such that the derived results have less decision variables. 
On the other hand, different from the discrete-time cases [3], [5], [7], [8], the application of the event-triggering mechanism for continuous-time systems could lead to a well-known yet undesirable phenomenon called rendezvous (Zeno) phenomenon that possesses a strong destructiveness for the system equipment. So, the second challenge would be how to construct a suitable event generator function to avoid such a rendezvous behavior. Although the rendezvous problem has been discussed in [6], [10] for multi-agent systems, the mathematical analysis problem on this behavior is still open for the state estimation issues of complex networks with time-varying delays. It is, therefore, the second motivation of this paper to provide satisfactory answers to the two questions mentioned above and also propose a design scheme of the event-based estimator for the addressed complex networks.

Motivated by the above discussions, in this paper, we aim to design an event-triggered estimator for a class of complex networks with time-varying delays. In terms of the Lyapunov functional approach combined with the comparison principle, some sufficient conditions are derived to guarantee that the error dynamics is exponentially ultimately bounded. Furthermore, the proposed results are specialized to two classes of complex networks. Finally, a numerical example is given to illustrate the effectiveness of the proposed event-based estimator. The novelties of this paper lie in the following three aspects: 1) a novel event generator function, which is dependent not only on the measurement output but also on a predefined positive constant, is proposed to reduce the communication burden; 2) the comparison principle is utilized to derive the desired sufficient conditions with the purpose of simplifying the estimator design; 3) a rigorous proof is proposed to show that the designed triggering condition is free of the Zeno behavior.

Notations: Throughout this paper, $\mathbb{R}^{n}$ denotes the $n$-dimensional Euclidean space. $\mathbb{N}$ denotes the set of natural numbers. For $x \in \mathbb{R}^{n}, x^{T}$ denotes its transpose. The vector norm is defined as $\|x\|=\sqrt{x^{T} x}$. $I_{n}$ denotes $n$ dimensional identity matrix. For matrix $A \in \mathbb{R}^{n \times n},\|A\|=\sqrt{\lambda_{\max }\left(A^{T} A\right)}$, where $\lambda_{\max }(\cdot)$ represents the largest eigenvalue. Moreover, for real symmetric matrices $X$ and $Y$, the notation $X \leq Y$ (respectively, $X<Y$ ) means that the matrix $X-Y$ is negative semidefinite (respectively, negative definite). $C\left([-r, 0] ; \mathbb{R}^{n}\right)$ denotes the family of continuous functions from $[-r, 0]$ to $\mathbb{R}^{n}$ with the norm $\|\phi\|_{r}=\sup _{-r \leq \theta \leq 0} \phi(\theta)$.

\section{PRoblem Formulation AND PRELIMINARIES}

In this paper, consider the following time-delayed complex network consisting of $N$ coupled nodes of the form:

$$
\left\{\begin{array}{l}
\dot{x}_{i}(t)=D x_{i}(t)+f\left(x_{i}(t)\right)+g\left(x_{i}(t-\tau(t))\right)+\sum_{j=1}^{N} a_{i j} \Gamma x_{j}(t) \\
y_{i}(t)=C_{i} x_{i}(t)
\end{array}\right.
$$

where $x_{i}(t) \in \mathbb{R}^{n}$ and $y_{i}(t) \in \mathbb{R}^{m}(i=1,2, \ldots, N)$ are, respectively, the state vector and the measurement output of the $i$ th node at time $t . D \in \mathbb{R}^{n \times n}$ and $C_{i} \in \mathbb{R}^{m \times n}$ are two known constant matrices. $f(\cdot)$ and $g(\cdot)$ are nonlinear functions satisfying certain conditions to be given later. $\tau(t)$ describes the known time-varying delay satisfying $0 \leq \tau(t) \leq \tau$, where $\tau$ is a known positive scalar representing the maximum delay. $\Gamma=\operatorname{diag}\left\{\gamma_{1}, \gamma_{2}, \ldots, \gamma_{n}\right\}$ is the inner-coupling matrix linking the $j$ th state variable as $\gamma_{i}>0(i=1,2, \ldots, n) . A=\left[a_{i j}\right]_{N \times N}$ is the coupled configuration matrix of the network with $a_{i j}>0(i \neq j)$ but not all zero. Furthermore, the diagonal element $a_{i i}$ satisfies

$$
a_{i i}=-\sum_{j=1, j \neq i}^{N} a_{i j}
$$

As discussed in the introduction, an event-triggered mechanism is adopted to reduce the communication burden. In order to characterize such a mechanism, let the triggering time sequence of node $i$ be

$$
t_{0}=t_{0}^{i}<t_{1}^{i}<\ldots t_{k}^{i}<\ldots
$$


For each node $i$, the sequence of event triggering instants is determined iteratively by

$$
t_{k+1}^{i}=\inf \left\{t \mid t>t_{k}^{i}, \breve{f}_{i}\left(y_{i}(t), \delta_{i}, \beta\right)>0\right\}
$$

where the event generator function $\breve{f}_{i}\left(y_{i}(t), \delta_{i}, \beta\right)$ to be defined later is dependent on the measurement output and the given thresholds $\beta>0$ and $\delta_{i}>0$.

In the event-triggered setup, the state estimator with zero-order holder on node $i$ is proposed as follows:

$$
\begin{aligned}
\dot{\hat{x}}_{i}(t)= & D \hat{x}_{i}(t)+f\left(\hat{x}_{i}(t)\right)+g\left(\hat{x}_{i}(t-\tau(t))\right) \\
& +\sum_{j=1}^{N} a_{i j} \Gamma \hat{x}_{j}(t)+K_{i}\left(y_{i}\left(t_{k}^{i}\right)-C_{i} \hat{x}_{i}\left(t_{k}^{i}\right)\right), \quad t \in\left[t_{k}^{i}, t_{k+1}^{i}\right)
\end{aligned}
$$

where $\hat{x}_{i}(t)$ is the estimate of the state $x_{i}(t)$ and $K_{i} \in \mathbb{R}^{n \times m}$ is the estimator gain matrix to be designed.

Now, for the implementation of designed estimators, we adopt the event generator function on node $i$ as follows:

$$
\breve{f}_{i}\left(y_{i}(t), \delta_{i}, \beta\right)=\varepsilon_{i}^{T}(t) \varepsilon_{i}(t)-\beta\left\|y_{i}(t)-C_{i} \hat{x}_{i}(t)\right\|^{2}-\delta_{i}
$$

where $\varepsilon_{i}(t)=y_{i}(t)-C_{i} \hat{x}_{i}(t)-\left(y_{i}\left(t_{k}^{i}\right)-C_{i} \hat{x}_{i}\left(t_{k}^{i}\right)\right)$ and $y_{i}\left(t_{k}^{i}\right)$ is the measurement output at the latest event instant.

Remark 1: In this paper, the selected triggering function (4) is linearly dependent on the measurement's norm and a predefined positive constant. It is not difficult to see that the triggering function (4) will reduce to the case in [15], [45] when $\beta=0$. In addition, the inter-event intervals for each node become shorter as $\delta_{i}$ decreases. Therefore, such a construction provides more design margin/flexibility to exclude the Zeno phenomenon.

Let $e_{i}(t)=\hat{x}_{i}(t)-x_{i}(t), \hat{f}\left(e_{i}(t)\right)=f\left(\hat{x}_{i}(t)\right)-f\left(x_{i}(t)\right)$ and $\hat{g}\left(e_{i}(t)\right)=g\left(\hat{x}_{i}(t)\right)-g\left(x_{i}(t)\right)$. It follows from (1) and (3) that the error dynamics of the state estimation obeys

$$
\dot{e}_{i}(t)=D e_{i}(t)+\hat{f}\left(e_{i}(t)\right)+\hat{g}\left(e_{i}(t-\tau(t))\right)+\sum_{j=1}^{N} a_{i j} \Gamma e_{j}(t)-K_{i} C_{i} e_{i}(t)+K_{i} \varepsilon_{i}(t) .
$$

For notational simplicity, we define

$$
\begin{aligned}
e(t) & =\left[e_{1}^{T}(t), e_{2}^{T}(t), \ldots, e_{N}^{T}(t)\right]^{T} \\
\varepsilon(t) & =\left[\varepsilon_{1}^{T}(t), \varepsilon_{2}^{T}(t), \ldots, \varepsilon_{N}^{T}(t)\right]^{T} \\
F(e(t)) & =\left[\hat{f}^{T}\left(e_{1}(t)\right), \hat{f}^{T}\left(e_{2}(t)\right), \ldots, \hat{f}^{T}\left(e_{N}(t)\right)\right]^{T} \\
G(e(t)) & =\left[\hat{g}^{T}\left(e_{1}(t)\right), \hat{g}^{T}\left(e_{2}(t)\right), \ldots, \hat{g}^{T}\left(e_{N}(t)\right)\right]^{T} \\
\mathbf{D} & =I_{N} \otimes D, \quad C=\operatorname{diag}\left\{C_{1}, C_{2} \ldots, C_{N}\right\} \\
K & =\operatorname{diag}\left\{K_{1}, K_{2} \ldots, K_{N}\right\} .
\end{aligned}
$$

By using the matrix's Kronecker product, the error dynamics (5) can be rewritten as

$$
\dot{e}(t)=\mathbf{D} e(t)+F(e(t))+G(e(t))+(A \otimes \Gamma) e(t)-K C e(t)+K \varepsilon(t) .
$$

Before proceeding further, we introduce the following definitions, lemmas and assumptions.

Definition 1 ( [45]): The dynamics of the estimation error is exponentially ultimately bounded if there exist positive constants $M, \lambda$ and $\omega$ such that

$$
\|e(t)\|^{2} \leq M e^{-\lambda\left(t-t_{0}\right)}+\omega .
$$


Assumption 1: For $\forall x, y \in \mathbb{R}^{n}$, the nonlinear vector-valued functions $f$ and $g$ are continuous and satisfy

$$
\begin{aligned}
& \|f(x)-f(y)\| \leq\left\|L_{1}\right\|\|x-y\| \\
& \|g(x)-g(y)\| \leq\left\|L_{2}\right\|\|x-y\|
\end{aligned}
$$

where $L_{1}$ and $L_{2}$ are known constant matrices with appropriate dimensions.

Lemma 1: For $\forall x, y \in \mathbb{R}^{n}$, the following inequality holds

$$
x^{T} y+y^{T} x \leq x^{T} Q x+y^{T} Q^{-1} y
$$

where $Q$ is a diagonal positive definite matrix with appropriate dimensional.

Lemma 2 ([13]): If $P \in \mathbb{R}^{n \times n}$ is a symmetric and positive definite matrix and $Q \in \mathbb{R}^{n \times n}$ is a symmetric matrix, then

$$
\lambda_{\min }\left(P^{-1} Q\right) x^{T} P x \leq x^{T} Q x \leq \lambda_{\max }\left(P^{-1} Q\right) x^{T} P x, \quad x \in \mathbb{R}^{n} .
$$

The main purpose of this paper is to design a state estimator such that the error dynamics (5) or (6) with the triggering time sequence given in (2) is exponentially ultimately bounded.

\section{MAIN RESULTS}

In this section, the stability of the error dynamics is firstly investigated by means of the comparison principle and Lyapunov functional approach. Then, a design scheme of the state estimator for addressed complex networks is given via the solution to certain matrix inequalities.

Theorem 1: Let the estimator gain matrix $K_{i}(i=1,2, \ldots, N)$ and the threshold parameters $\beta$ and $\delta_{i}(i=$ $1,2, \ldots, N)$ be given. If there exist a positive definite matrix $P \in \mathbb{R}^{n N \times n N}$, two diagonal positive definite matrices $Q, S \in \mathbb{R}^{n \times n}$ and two positive scalars $\mu$ and $\rho$ satisfying the following condition

$$
\begin{aligned}
P \mathbf{D} & +\mathbf{D}^{T} P-P K C-C^{T} K^{T} P+P(A \otimes \Gamma)+(A \otimes \Gamma)^{T} P \\
& +P\left(Q \otimes I_{N}\right)^{-1} P+P\left(S \otimes I_{N}\right)^{-1} P+\left\|L_{1}\right\|^{2}\left(Q \otimes I_{N}\right)+\mu P+\frac{P K K^{T} P}{\rho}<0, \\
& -\mu+\eta+\gamma<0,
\end{aligned}
$$

then the error dynamics (5) or (6) with triggering time sequences (2) is exponentially ultimately bounded where

$$
\eta=\lambda_{\max }\left(P^{-1}\left\|L_{2}\right\|^{2}\left(S \otimes I_{N}\right)\right), \quad \gamma=\max _{i=1,2, \ldots, N}\left\{\frac{\rho \beta\left\|C_{i}\right\|^{2}}{\lambda_{\min }(P)}\right\} .
$$

Proof: First, consider the following Lyapunov function

$$
V(t)=e^{T}(t) P e(t) .
$$

Then, along the trajectory of system (6), calculating the derivative of $V(t)$ leads to

$$
\dot{V}(t)=2 e^{T}(t) P[\mathbf{D} e(t)+F(e(t))+G(e(t-\tau(t)))+(A \otimes \Gamma) e(t)-K C e(t)+K \varepsilon(t)] .
$$

In what follows, by utilizing Lemmas 1 and 2, we have

$$
\begin{aligned}
2 e^{T}(t) P F(e(t)) \leq & e^{T}(t) P\left(Q \otimes I_{N}\right)^{-1} P e(t)+\left\|L_{1}\right\|^{2} e^{T}(t)\left(Q \otimes I_{N}\right) e(t) \\
2 e^{T}(t) P G(e(t-\tau(t))) \leq & e^{T}(t) P\left(S \otimes I_{N}\right)^{-1} P e(t) \\
& +\lambda_{\max }\left(P^{-1}\left\|L_{2}\right\|^{2} S \otimes I_{N}\right) e^{T}(t-\tau(t)) P e(t-\tau(t)) \\
2 e^{T}(t) P K \varepsilon(t) \leq & \frac{e^{T}(t) P K K^{T} P e(t)}{\rho}+\rho \varepsilon(t)^{T} \varepsilon(t) .
\end{aligned}
$$


In view of (11), (14)-(17), one has

$$
\dot{V}(t) \leq-\mu V(t)+\eta V(t-\tau(t))+\rho \sum_{i=1}^{N} \varepsilon_{i}(t)^{T} \varepsilon_{i}(t), \quad t \in\left[t_{k}^{i}, t_{k+1}^{i}\right) .
$$

On the other hand, as $t \in\left[t_{k}^{i}, t_{k+1}^{i}\right)$, the triggering condition (2) can be rewritten as

$$
\varepsilon_{i}^{T}(t) \varepsilon_{i}(t) \leq \beta\left\|C_{i}\right\|^{2}\left\|e_{i}(t)\right\|^{2}+\delta_{i}
$$

which implies

$$
\varepsilon^{T}(t) \varepsilon(t) \leq \beta \max _{i=1,2, \ldots, N}\left\{\left\|C_{i}\right\|^{2}\right\} \frac{1}{\lambda_{\min }(P)} e^{T}(t) P e(t)+\sum_{i=1}^{N} \delta_{i} .
$$

Letting $\Delta=\rho \sum_{i=1}^{N} \delta_{i}$ and taking the above inequality into account, we obtain from (18) that

$$
\dot{V}(t) \leq(-\mu+\gamma) V(t)+\eta V(t-\tau(t))+\Delta .
$$

Now, let us investigate the exponentially ultimately boundedness of the solution of the differential equation governing the error dynamics by using the comparison principle. For this purpose, let $v(t)$ be a unique solution of the following delayed system

$$
\left\{\begin{array}{l}
\dot{v}(t)=(-\mu+\gamma) v(t)+\eta v(t-\tau(t))+\Delta+\epsilon \\
v(s)=V(s), \quad s \in[-\tau, 0]
\end{array}\right.
$$

where $\epsilon$ is a positive scalar. Then, by using the formula of integration, system (22) yields

$$
v(t) \leq M e^{(-\mu+\gamma)\left(t-t_{0}\right)}+\int_{t_{0}}^{t} e^{(-\mu+\gamma)(t-s)}[\eta v(s-\tau(s))+\Delta+\epsilon] d s
$$

where $M=\sup _{-\tau \leq t \leq 0} v(t)$. Therefore, we only need to prove

$$
v(t)<M e^{-\lambda\left(t-t_{0}\right)}+\frac{\epsilon+\Delta}{\mu-\gamma-\eta}
$$

where $\lambda>0$ is the unique solution of

$$
\lambda-\mu+\gamma+\eta e^{\lambda \tau}=0
$$

Furthermore, the proof can be divided into two parts, that is, the part of $t \in\left[t_{0}-\tau, t_{0}\right]$ and the part $t>t_{0}$.

The part of $t \in\left[t_{0}-\tau, t_{0}\right]:$ It is easy to see that

$$
v(t) \leq M \leq M e^{-\lambda\left(t-t_{0}\right)}<M e^{-\lambda\left(t-t_{0}\right)}+\frac{\epsilon+\Delta}{\mu-\gamma-\eta} .
$$

The part of $t>t_{0}$ : In this part, the proof is carried out by using contradiction. Specially, assume that there exists at least one $t>t_{0}$ such that

$$
v(t) \geq M e^{-\lambda\left(t-t_{0}\right)}+\frac{\epsilon+\Delta}{\mu-\gamma-\eta} .
$$

Denote $t^{*}=\inf \left\{t>t_{0} \mid v(t) \geq M e^{-\lambda\left(t-t_{0}\right)}+\frac{\epsilon+\Delta}{\mu-\gamma-\eta}\right\}$. In the following, we shall use this $t^{*}$ to reveal that (27) is not true.

First, according to the definition of $t^{*}$, one has

$$
v\left(t^{*}\right)=M e^{-\lambda\left(t^{*}-t_{0}\right)}+\frac{\epsilon+\Delta}{\mu-\gamma-\eta}
$$


and

$$
v(t)<M e^{-\lambda\left(t-t_{0}\right)}+\frac{\epsilon+\Delta}{\mu-\gamma-\eta}, \quad t<t^{*}
$$

Then, it follows from (23) that

$$
\begin{aligned}
v\left(t^{*}\right)= & M e^{(-\mu+\gamma)\left(t^{*}-t_{0}\right)}+\int_{t_{0}}^{t^{*}} e^{(-\mu+\gamma)\left(t^{*}-s\right)}[\eta v(s-\tau(s))+\Delta+\epsilon] d s \\
< & e^{(-\mu+\gamma)\left(t^{*}-t_{0}\right)}\left\{M+\frac{\epsilon+\Delta}{\mu-\gamma-\eta}+\int_{t_{0}}^{t^{*}} e^{(\mu-\gamma)\left(s-t_{0}\right)}\right. \\
& \left.\times\left[\eta\left(M e^{-\lambda\left(s-\tau(s)-t_{0}\right)}+\frac{\epsilon+\Delta}{\mu-\gamma-\eta}\right)+\Delta+\epsilon\right] d s\right\} \\
< & e^{(-\mu+\gamma)\left(t^{*}-t_{0}\right)}\left\{M+\frac{\epsilon+\Delta}{\mu-\gamma-\eta}+M \eta e^{\lambda \tau} \int_{t_{0}}^{t^{*}} e^{(\mu-\gamma-\lambda)\left(s-t_{0}\right)} d s\right. \\
& \left.+\frac{(\mu-\gamma)(\epsilon+\Delta)}{\mu-\gamma-\eta} \int_{t_{0}}^{t^{*}} e^{(\mu-\gamma)\left(s-t_{0}\right)} d s\right\} \\
= & M e^{-\lambda\left(t^{*}-t_{0}\right)}+\frac{\epsilon+\Delta}{\mu-\gamma-\eta},
\end{aligned}
$$

which contradicts to (27) or (28) and therefore

$$
v(t)<M e^{-\lambda\left(t-t_{0}\right)}+\frac{\epsilon+\Delta}{\mu-\gamma-\eta} .
$$

By means of the comparison principle [42], we have

$$
V(t) \leq v(t)<M e^{-\lambda\left(t-t_{0}\right)}+\frac{\epsilon+\Delta}{\mu-\gamma-\eta}
$$

which, as $\epsilon \rightarrow 0$, results in

$$
V(t) \leq M e^{-\lambda\left(t-t_{0}\right)}+\frac{\Delta}{\mu-\gamma-\eta} .
$$

Finally, we will prove that the Zeno behavior can be excluded for the selected triggering condition. For $t \in$ $\left[t_{k}^{i}, t_{k+1}^{i}\right)$, computing the upper right-hand Dini derivative of $\left\|\varepsilon_{i}(t)\right\|$ yields

$$
\begin{aligned}
D^{+}\left\|\varepsilon_{i}(t)\right\| \leq & \left\|\dot{\varepsilon}_{i}(t)\right\| \\
\leq & \left\|C_{i}\right\|\left[\left\|\mathbf{D}-K_{i} C_{i}\right\|\left\|e_{i}(t)\right\|+\left\|L_{1}\right\|\left\|e_{i}(t)\right\|\right. \\
& \left.+\left\|L_{2}\right\| e_{i}(t-\tau(t))\|+\| \sum_{j=1}^{N} a_{i j} \Gamma e_{j}(t)\|+\| K_{i}\|\| \varepsilon_{i}(t) \|\right] .
\end{aligned}
$$

For the three variables on the right side of above inequality, we have

$$
\begin{aligned}
\left\|e_{i}(t)\right\| & \leq \sqrt{\frac{M}{\lambda_{\min }(P)}} e^{-\frac{\lambda}{2}\left(t-t_{0}\right)}+\sqrt{\frac{\Delta}{\lambda_{\min }(P)(\mu-\gamma-\eta)}} \\
& \leq \sqrt{\frac{M}{\lambda_{\min }(P)}} e^{-\frac{\lambda}{2}\left(t_{k}^{i}-t_{0}\right)}+\sqrt{\frac{\Delta}{\lambda_{\min }(P)(\mu-\gamma-\eta)}} \\
\left\|\sum_{j=1}^{N} a_{i j} \Gamma e_{j}(t)\right\| & \leq\left|a_{i i}\right|\|\Gamma\| \sum_{j=1}^{N}\left\|e_{j}(t)\right\| \leq\left|a_{i i}\right|\|\Gamma\|\left[\sqrt{\frac{N M}{\lambda_{\min }(P)}} e^{-\frac{\lambda}{2}\left(t_{k}^{i}-t_{0}\right)}+\sqrt{\frac{\Delta}{\lambda_{\min }(P)(\mu-\gamma-\eta)}}\right] \\
\left\|e_{i}(t-\tau(t))\right\| & \leq \sqrt{\frac{M}{\lambda_{\min }(P)}} e^{\frac{\lambda}{2} \tau} e^{-\frac{\lambda}{2}\left(t_{k}^{i}-t_{0}\right)}+\sqrt{\frac{\Delta}{\lambda_{\min }(P)(\mu-\gamma-\eta)}} .
\end{aligned}
$$


Taking (35) into (34) leads to

$$
\begin{aligned}
D^{+}\left\|\varepsilon_{i}(t)\right\| \leq & \left\|C_{i}\right\|\left[\left(\left\|D-K_{i} C_{i}\right\|+\left\|L_{1}\right\|\right)\left(\sqrt{\frac{M}{\lambda_{\min }(P)}} e^{-\frac{\lambda}{2}\left(t_{k}^{i}-t_{0}\right)}+\sqrt{\frac{\Delta}{\lambda_{\min }(P)(\mu-\gamma-\eta)}}\right)\right. \\
& +\left\|L_{2}\right\|\left(\sqrt{\frac{M}{\lambda_{\min }(P)}} e^{\frac{\lambda}{2} \tau} e^{-\frac{\lambda}{2}\left(t_{k}^{i}-t_{0}\right)}+\sqrt{\frac{\Delta}{\lambda_{\min }(P)(\mu-\gamma-\eta)}}\right) \\
& \left.+\left|a_{i i}\right|\|\Gamma\|\left(\sqrt{\frac{N M}{\lambda_{\min }(P)}} e^{-\frac{\lambda}{2}\left(t_{k}^{i}-t_{0}\right)}+\sqrt{\frac{\Delta}{\lambda_{\min }(P)(\mu-\gamma-\eta)}}\right)+\left\|C_{i}\right\|\left\|K_{i}\right\|\left\|\varepsilon_{i}(t)\right\|\right] .
\end{aligned}
$$

Denoting

$$
\begin{aligned}
\alpha_{t_{k}^{i}}= & \left\|C_{i}\right\|\left[\left(\left\|D-K_{i} C_{i}\right\|+\left\|L_{1}\right\|\right)\left(\sqrt{\frac{M}{\lambda_{\min }(P)}} e^{-\frac{\lambda}{2}\left(t_{k}^{i}-t_{0}\right)}+\sqrt{\frac{\Delta}{\lambda_{\min }(P)(\mu-\gamma-\eta)}}\right)\right. \\
& +\left\|L_{2}\right\|\left(\sqrt{\frac{M}{\lambda_{\min }(P)}} e^{\frac{\lambda}{2} \tau} e^{-\frac{\lambda}{2}\left(t_{k}^{i}-t_{0}\right)}+\sqrt{\frac{\Delta}{\lambda_{\min }(P)(\mu-\gamma-\eta)}}\right) \\
& \left.+\left|a_{i i}\right|\|\Gamma\|\left(\sqrt{\frac{N M}{\lambda_{\min }(P)}} e^{-\frac{\lambda}{2}\left(t_{k}^{i}-t_{0}\right)}+\sqrt{\frac{\Delta}{\lambda_{\min }(P)(\mu-\gamma-\eta)}}\right)\right],
\end{aligned}
$$

we can find from (36) that

$$
\left\|\varepsilon_{i}(t)\right\| \leq \frac{\alpha_{t_{k}^{i}}\left(e^{\left\|K_{i}\right\|\left\|C_{i}\right\|\left(t-t_{k}^{i}\right)}-1\right)}{\left\|K_{i}\right\|\left\|C_{i}\right\|} .
$$

In addition, we have

$$
\left\|\varepsilon_{i}\left(t_{k+1}^{i}\right)\right\| \leq \frac{\alpha_{t_{k}^{i}}\left(e^{\left\|K_{i}\right\|\left\|C_{i}\right\|\left(t_{k+1}^{i}-t_{k}^{i}\right)}-1\right)}{\left\|K_{i}\right\|\left\|C_{i}\right\|}
$$

Note that the next event will not be triggered before $\left\|\varepsilon_{i}(t)\right\|=\delta_{i}$. Thus

$$
t_{k+1}^{i}-t_{k}^{i} \geq \ln \left(\frac{\delta_{i}\left\|K_{i}\right\|\left\|C_{i}\right\|}{\alpha_{t_{k}^{i}}}+1\right)>0,
$$

which completes the proof.

Remark 2: As we know, the Zeno phenomenon resulting from the application of the event-triggering mechanism is a typical dynamical behavior which prevents the implementation of sampling devices. Up to now, some preliminary results have been reported in the literature on the Zeno problem (see e.g. [6], [10]). In this paper, by utilizing the Dini derivative, the lower bound of the interval times is accurately given. It is worth mentioning that this interval can be obtained via distributed computation as it is dependent on the selected parameter $\delta_{i}$ and the designed estimator gain $K_{i}$. Due to the existence of $\delta_{i}$, the exponentially ultimately bound of the estimation error is investigated. However, the results in this paper can be easily extended to the exponentially convergence by designing the similar event-triggering sequence $f_{i}$ as (10) in [18].

Remark 3: Traditionally, for networks with time-varying delays, the Lyapunov-Krasovskii functional approach has been widely utilized to analyze the system performance, and this would inevitably lead to more decision variables and more conservative sufficient conditions. For instance, in [45], the event-triggering state estimation problem was investigated for complex networks with mixed time delays, where the Lyapunov-Krasovskii functional approach was used to obtain the main results. It can be seen from Theorem 1 that in [45], the decision variables are $3 N n(N n+1)+2(N n)^{2}+11$, where $N$ is the number of the nodes and $n$ is the dimensional size of each node, while the decision variables in Theorem 1 are $N n(N n+1) / 2+n(n+1)+2$. Thus, the complexity of LMI 
computations in [45] remains higher than that of our results. Accordingly, the resulting design approach would have limited application scope in real-world problems. Different from traditional approaches, in this paper, the comparison principle is skillfully to derive a simplified matrix inequality whose feasibility guarantees the exponentially ultimately boundedness of the addressed complex networks with both the event-triggering mechanism and the time-varying delays.

Up to now, the analysis problem of the estimator performance has been dealt with. Next, we are in a position to consider the estimator design problem for the complex network (1). The following result can be easily accessible from Theorem 1 and the proof is therefore omitted.

Theorem 2: Let the threshold parameters $\beta$ and $\delta_{i}(i=1,2, \ldots, N)$ be given. If there exist a matrix $Y=$ $\operatorname{diag}\left\{Y_{1}, Y_{2}, \ldots, Y_{N}\right\} \in \mathbb{R}^{N n \times N m}$, a positive definite matrix $P=\operatorname{diag}\left\{P_{1}, P_{2}, \ldots, P_{N}\right\} \in \mathbb{R}^{n N \times n N}$, two diagonal positive definite matrices $Q, S \in \mathbb{R}^{n \times n}$, and two positive scalars $\mu$ and $\rho$ satisfying the following condition

$$
\begin{aligned}
P \mathbf{D} & +\mathbf{D}^{T} P-Y C-C^{T} Y^{T}+P(A \otimes \Gamma)+(A \otimes \Gamma)^{T} P \\
& \quad+P\left(Q \otimes I_{N}\right)^{-1} P+P\left(S \otimes I_{N}\right)^{-1} P+\left\|L_{1}\right\|^{2}\left(Q \otimes I_{N}\right)+\mu P+\frac{Y Y^{T}}{\rho}<0 \\
& -\mu+\eta+\gamma<0
\end{aligned}
$$

then the error dynamics (5) or (6) with triggering time sequences (2) is exponentially ultimately bounded. Furthermore, if (40) and (41) hold, then the estimator gain matrix on node $i$ is given by $K_{i}=P_{i}^{-1} Y_{i}$.

In what follows, we show that our main result can be easily specialized to the following two cases: 1) the complex networks without time-delays; and 2) the complex networks with $N=1$ (i.e. general nonlinear systems).

Case 1: The complex networks without time-delays.

In this case, the complex network (1) reduces to

$$
\left\{\begin{array}{l}
\dot{x}_{i}(t)=D x_{i}(t)+f\left(x_{i}(t)\right)+\sum_{j=1}^{N} a_{i j} \Gamma x_{j}(t) \\
y_{i}(t)=C_{i} x_{i}(t)
\end{array}\right.
$$

and the corresponding state estimator is of the form

$$
\dot{\hat{x}}_{i}(t)=D \hat{x}_{i}(t)+f\left(\hat{x}_{i}(t)\right)+\sum_{j=1}^{N} a_{i j} \Gamma \hat{x}_{j}(t)+K_{i}\left(y_{i}\left(t_{k}^{i}\right)-C_{i} \hat{x}_{i}\left(t_{k}^{i}\right)\right), \quad i=1,2, \ldots, N .
$$

According to (42) and (43), the error dynamics becomes

$$
\dot{e}_{i}(t)=D e_{i}(t)+\hat{f}\left(e_{i}(t)\right)+\sum_{j=1}^{N} a_{i j} \Gamma e_{j}(t)-K_{i} C_{i} e_{i}(t)+K_{i} \varepsilon_{i}(t) .
$$

Furthermore, by means of Theorem 2, we have the following corollary readily.

Corollary 1: Let the threshold parameters $\beta$ and $\delta_{i}(i=1,2, \ldots, N)$ be given. If there exist a matrix $\bar{Y}=$ $\operatorname{diag}\left\{\bar{Y}_{1}, \bar{Y}_{2}, \ldots, \bar{Y}_{N}\right\} \in \mathbb{R}^{N n \times N m}$, a positive definite matrix $\bar{P}=\operatorname{diag}\left\{\bar{P}_{1}, \bar{P}_{2}, \ldots, \bar{P}_{N}\right\} \in \mathbb{R}^{n N \times n N}$, a diagonal positive definite matrix $\bar{Q} \in \mathbb{R}^{n \times n}$, and two positive scalars $\bar{\mu}$ and $\bar{\rho}$ satisfying the following condition:

$$
\begin{aligned}
& \bar{P} \mathbf{D}+\mathbf{D}^{T} \bar{P}-\bar{Y} C-C^{T} \bar{Y}^{T}+\bar{P}(A \otimes \Gamma)+(A \otimes \Gamma)^{T} \bar{P} \\
& \quad+\bar{P}\left(\bar{Q} \otimes I_{N}\right)^{-1} \bar{P}+\left\|L_{1}\right\|^{2}\left(\bar{Q} \otimes I_{N}\right)+\bar{\mu} \bar{P}+\frac{\bar{Y} \bar{Y}^{T}}{\bar{\rho}}<0 \\
& -\bar{\mu}+\bar{\gamma}<0
\end{aligned}
$$


with $\bar{\gamma}=\max _{i=1,2, \ldots, N}\left\{\frac{\rho \beta\left\|C_{i}\right\|^{2}}{\lambda_{\min }(P)}\right\}$, then the error dynamics (44) with triggering time sequences (2) is exponentially ultimately bounded. Furthermore, if (45) and (46) hold, then the estimator gain matrix on node $i$ is given by $K_{i}=\bar{P}_{i}^{-1} \bar{Y}_{i}$.

Case 2: The complex networks with $N=1$, i.e. general nonlinear systems.

As $N=1$, the complex network system (1) becomes the following nonlinear system with time-varying delays

$$
\left\{\begin{array}{l}
\dot{x}_{1}(t)=D x_{1}(t)+f\left(x_{1}(t)\right)+g\left(x_{1}(t-\tau(t))\right) \\
y_{1}(t)=C_{1} x_{1}(t) .
\end{array}\right.
$$

Similar to (3) and (5), the state estimator and error dynamics are, respectively, rewritten as follows

$$
\dot{\hat{x}}_{1}(t)=D \hat{x}_{1}(t)+f\left(\hat{x}_{1}(t)\right)+g\left(\hat{x}_{1}(t-\tau(t))\right)+\bar{K}_{1}\left(y_{1}\left(t_{k}^{1}\right)-C \hat{x}_{1}\left(t_{k}^{1}\right)\right)
$$

and

$$
\dot{e}_{1}(t)=D e_{1}(t)+\hat{f}\left(e_{1}(t)\right)-K_{1} C_{1} e_{1}(t)+K_{1} \varepsilon_{1}(t) .
$$

The following corollary can be obtained easily from Theorem 2 .

Corollary 2: Let the threshold parameters $\beta$ and $\delta_{1}$ in (2) be given. If there exist a matrix $\tilde{Y} \in \mathbb{R}^{m \times n}$, a positive definite matrix $\tilde{P} \in \mathbb{R}^{n \times n}$, two diagonal positive definite matrices $\tilde{Q}, \tilde{S} \in \mathbb{R}^{n \times n}$, and two positive scalars $\tilde{\mu}$ and $\tilde{\rho}$ satisfying the following condition:

$$
\begin{aligned}
\tilde{P} D & +D^{T} \tilde{P}-\tilde{Y} C_{1}-C_{1}^{T} \tilde{Y}^{T}+\tilde{P} \tilde{Q}^{-1} \tilde{P} \\
& +\tilde{P} \tilde{S}^{-1} \tilde{P}+\left\|L_{1}\right\|^{2} \tilde{Q}+\tilde{\mu} \tilde{P}+\frac{\tilde{Y} \tilde{Y}^{T}}{\tilde{\rho}}<0 \\
-\tilde{\mu} & +\tilde{\eta}+\tilde{\gamma}<0
\end{aligned}
$$

with $\tilde{\eta}=\lambda_{\max }\left(\tilde{P}^{-1}\left\|L_{2}\right\|^{2} \tilde{S}\right)$ and $\tilde{\gamma}=\max _{i=1,2, \ldots, N}\left\{\frac{\rho \beta\left\|C_{1}\right\|^{2}}{\lambda_{\min }(\tilde{P})}\right\}$, then the error dynamics (49) with triggering time sequences (2) is exponentially ultimately bounded. Furthermore, if (50) and (51) hold, then the estimator gain matrix is given by $K_{1}=\tilde{P}^{-1} \tilde{Y}$.

Remark 4: In this paper, the state estimation problem is investigated for a class of complex networks with time-varying delays and event-triggering communication protocol. By means of the constructed triggering function combined with the comparison principle, a novel scheme of estimator design is proposed to guarantee the desired estimation performance and exclude the undesired Zeno behavior. In addition, from Corollary 2, we can see that our results can also shed light on state estimation of general nonlinear time-delayed systems with event-triggering mechanism.

\section{NUMERICAl Simulations}

Consider the time-delayed complex network (1) with three nodes. The coupling configuration matrix is assumed to be

$$
A=\left[\begin{array}{ccc}
-2 & 1 & 1 \\
1 & -2 & 1 \\
1 & 1 & -2
\end{array}\right]
$$

The system matrix $D$, the inner-coupling matrix $\Gamma$ and the measurement matrices $C_{i}(i=1,2,3)$ are selected as

$$
D=-\left[\begin{array}{ll}
1 & 0 \\
0 & 1
\end{array}\right], \Gamma=\left[\begin{array}{ll}
1 & 0 \\
0 & 1
\end{array}\right], C_{i}=\left[\begin{array}{ll}
0.5 & 0.4
\end{array}\right]
$$


The nonlinear vector-valued functions $f\left(x_{i}(t)\right)$ and $g\left(x_{i}(t)\right)(i=1,2,3)$ are chosen as

$$
\begin{aligned}
& \left.f\left(x_{i}(t)\right)=\left[\begin{array}{cc}
0.5 & -0.2 \\
0.5 & 0.4
\end{array}\right]\left[\frac{\left|x_{i 1}(t)+1\right|-\left|x_{i 1}(t)-1\right|}{2}\right] \frac{\left|x_{i 2}(t)+1\right|-\left|x_{i 2}(t)-1\right|}{2}\right] \\
& g\left(x_{i}(t)\right)=\left[\begin{array}{cc}
0.7 & 0.3 \\
-0.1 & 0.4
\end{array}\right]\left[\frac{\left|x_{i 1}(t)+1\right|-\left|x_{i 1}(t)-1\right|}{2}\right]
\end{aligned}
$$

We can easily check that Assumption 1 is satisfied with

$$
L_{1}=\left[\begin{array}{cc}
0.5 & -0.2 \\
0.5 & 0.4
\end{array}\right], \quad L_{2}=\left[\begin{array}{cc}
0.7 & 0.3 \\
-0.1 & 0.4
\end{array}\right]
$$

Furthermore, in this example, the thresholds are selected as $\delta_{i}=0.6(i=1,2,3)$ and $\beta=0.2$. The time-varying delay is $\tau(t)=\frac{e^{t}}{1+e^{t}}$.

Using the Matlab software, a set of estimator gains in terms of the solution of Theorem 2 can be obtained as follows

$$
K_{1}=\left[\begin{array}{l}
0.9557 \\
0.9510
\end{array}\right], K_{2}=\left[\begin{array}{l}
0.7013 \\
0.7433
\end{array}\right], K_{3}=\left[\begin{array}{l}
0.7013 \\
0.7433
\end{array}\right] .
$$

Simulation results are shown in Figs. 1-3 for three nodes, and event-triggering instants for different $\delta_{i}$ are plotted in Fig. 4. It can be seen from Fig. 4 that a large $\delta_{i}$ can reduce the triggering frequency and thereby effectively alleviating the unnecessary energy consumption, while a small $\delta_{i}$ means a high triggering frequency and possibly results in a better system performance. We can see from the figures that the proposed estimator can provide satisfactory estimation performance for the addressed complex networks with event-triggering communication protocol. If we assume that $\delta=0.8$, then event-triggering instants for different $\beta$ are plotted in Fig. 5. It can be concluded that a small $\beta$ can make the conditions more feasible. Meanwhile, it will lead to frequently updates of the event-triggering mechanism, which means that more updates of the event-triggering mechanism are required to enforce the estimation error of (5) to zero.

\section{CONCLusion}

In this paper, the state estimation problem has been investigated for a class of complex networks with time-varying delays and event-triggering communication protocols. A new event-triggering function, in which the threshold is not only dependent on the measurement outputs but also dependent on a predefined constant, has been proposed to effectively reduce communication burden and avoid the Zeno phenomenon. By means of comparison principle and the Lyapunov function method, some sufficient conditions have derived to ensure that the estimation error dynamics is exponentially ultimately bounded. In addition, the desired estimator gains have been obtained in terms of the solution of matrix inequalities. Finally, a simulation has been given to illustrate the effectiveness of our theoretical results. Further research topics include the extension of this work to more general systems such as Markovian jumping systems [20], [30], [40], discrete-time systems and sensor networks [9], [22], [23].

\section{REFERENCES}

[1] A. Barabási and R. Albert, "Emergence of scaling in random networks," Science, vol. 286, pp. 509-512, 1999.

[2] H. Chen, J. Liang and Z. Wang, Pinning controllability of autonomous Boolean control networks, Science China Information Sciences, Vol. 59, No. 7, Jul. 2016, Art. No. 070107, DOI: 10.1007/s11432-016-5579-8.

[3] D. Ding, Z. Wang, and B. Shen, "Event-triggered consensus control for discrete-time stochastic multi-agent systems: The input-to-state stability in probability," Automatica, vol. 62, pp. 284-291, 2015. 


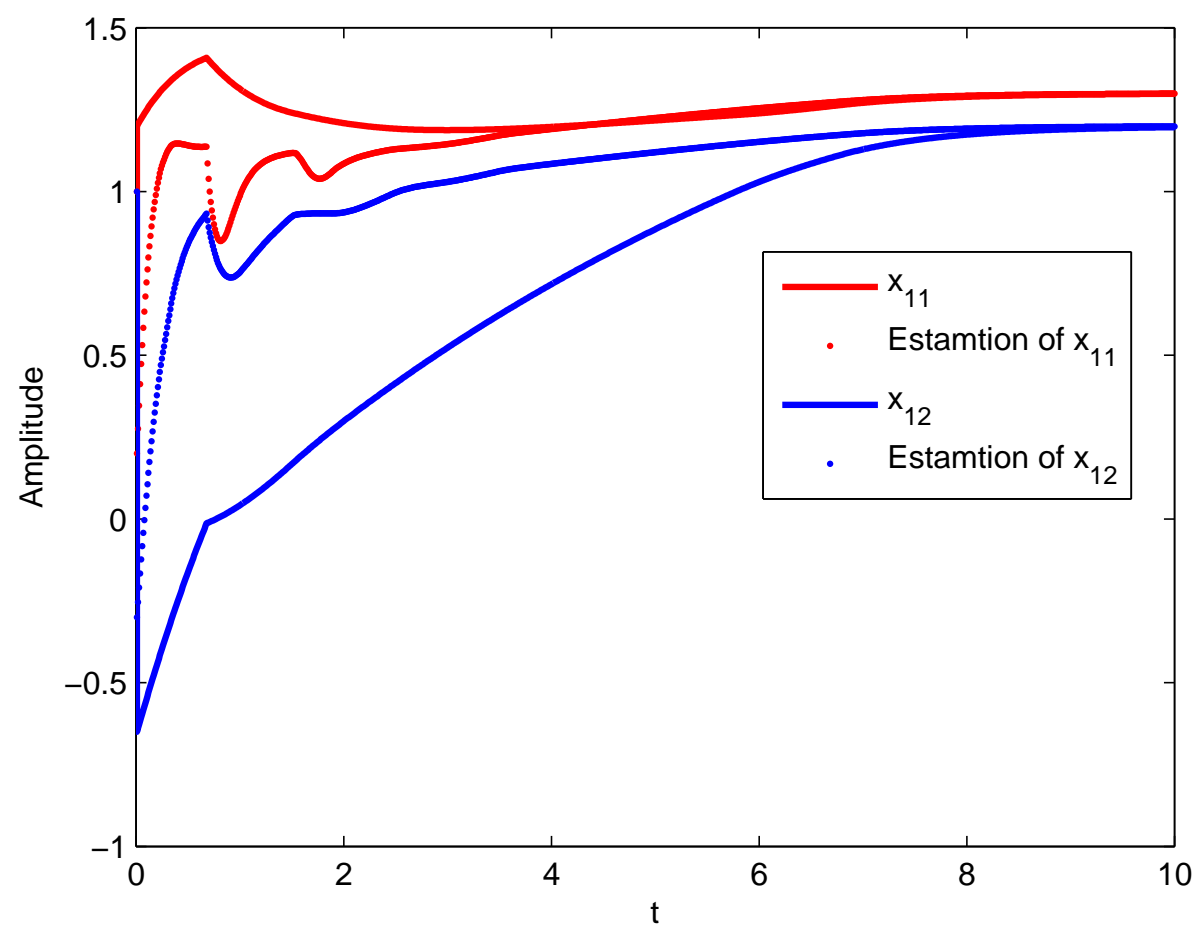

Fig. 1. State trajectories of $x_{1}$ and $\hat{x}_{1}$

[4] D. Ding, Z. Wang, B. Shen, and H. Shu, " $H_{\infty}$ state estimation for discrete-time complex networks with randomly occurring sensor saturations and randomly varying sensor delays," IEEE Transactions on Neural Networks and Learning Systems, vol. 23, pp. 725-736, 2012.

[5] D. Ding, Z. Wang, and B. Shen, "Event-triggered distributed $H_{\infty}$ state estimation with packet dropouts through sensor networks," IET Control Theory and Applications, vol. 9, no. 13, pp. 1948-1955, 2015.

[6] D. V. Dimarogonas, E. Frazzoli, and K. H. Johansson, "Distributed event-triggered control for multi-agent systems," IEEE Transactions on Automatic Control, vol. 57, no. 5, pp. 1291-1297, 2012.

[7] H. Dong, Z. Wang, S. X. Ding, and H. Gao, "Event-based $H_{\infty}$ filter design for a class of nonlinear time-varying systems with fading channels and multiplicative noises," IEEE Transactions on Signal Processing, vol. 63, no. 13, pp. 3387-3395, 2015.

[8] H. Dong, Z. Wang, B. Shen and D. Ding, Variance-constrained $H_{\infty}$ control for a class of nonlinear stochastic discrete time-varying systems: the event-triggered design, Automatica, Vol. 72, pp. 28-36, Oct. 2016.

[9] H. Dong, Z. Wang, X. Bu and F. E. Alsaadi, Distributed fault estimation with randomly occurring uncertainties over sensor networks, International Journal of General Systems, Vol. 45, No. 5, Jul. 2016, pp. 662-674.

[10] Y. Fan, L. Liu, G. Feng, and Y. Wang, "Self-triggered consensus for multi-agent systems with zeno-free triggers," IEEE Transactions on Automatic Control, vol. 60, no. 10, pp. 2779-2784, 2015.

[11] Z. Fei, H. Gao, and W. X. Zheng, "New synchronization stability of complex networks with an interval time-varying coupling delay," IEEE Transactions on Circuits and Systems II, vol. 56, no. 6, pp. 499-503, 2009.

[12] L. Gao, X. Liao, and H. Li, "Pinning controllability analysis of complex networks with a distributed event-triggered mechanism," IEEE transactions on circuits and systems - II: Express Briefs, vol. 61, pp. 541-545, 2014.

[13] Z. H. Guan, D. J. Hill, and X. Shen, "On hybrid impulsive and switching systems and application to nonlinear control," IEEE Trans. Automatic Control, vol. 50, pp. 1058-1062, 2005.

[14] N. Hou, H. Dong, Z. Wang, W. Ren and F. E. Alsaadi, Non-fragile state estimation for discrete Markovian jumping neural networks, Neurocomputing, Vol. 179, pp. 238-245, Feb. 2016.

[15] J. Hu, Z. Wang, J. Liang, and H. Dong, "Event-triggered distributed state estimation with randomly occurring uncertainties and nonlinearities over sensor networks: a delay-fractioning approach,” Journal of the Franklin Institute, Vol. 352, No. 9, pp. 3750-3763, 2015. 


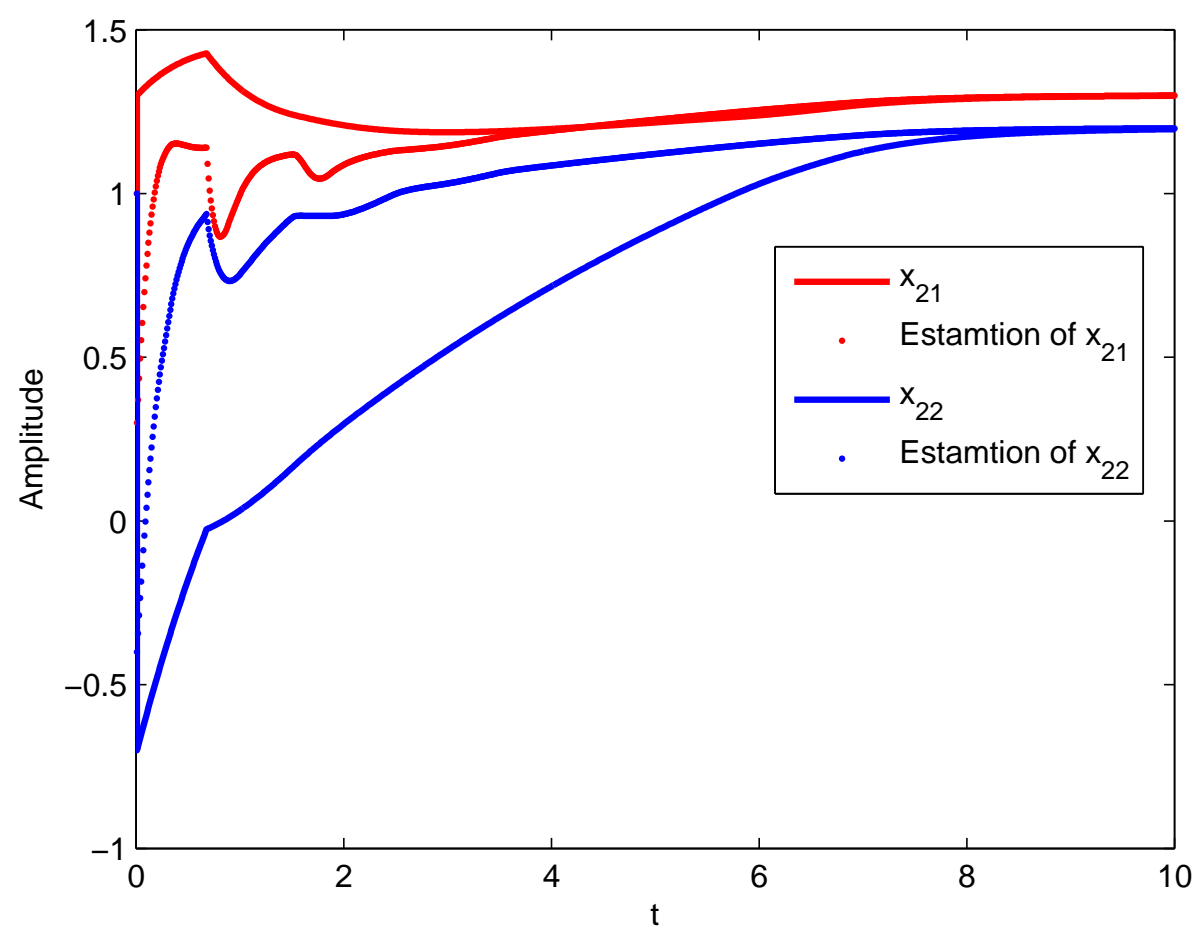

Fig. 2. State trajectories of $x_{2}$ and $\hat{x}_{2}$

[16] H. R. Karimi, and H. Gao, New Delay-Dependent Exponential $H_{\text {infty }}$ Synchronization for Uncertain Neural Networks With Mixed Time Delays, IEEE Transactions on Systems, Man, and Cybernetics, Part B (Cybernetics), vol. 40, pp. 173-185, 2010.

[17] H. Liu, Z. Wang, B. Shen and F. E. Alsaadi, State estimation for discrete-time memristive recurrent neural networks with stochastic time-delays, International Journal of General Systems, Vol. 45, No. 5, Jul. 2016, pp. 633-647.

[18] L. Li, D. W. C. Ho, J. Cao, and J. Lu, Pinning cluster synchronization in an array of coupled neural networks under event-based mechanism, Neural Networks, vol. 76, pp. 1-12, 2016.

[19] L. Li, D. W. C. Ho, and S. Xu, A distributed event-triggered scheme for discrete-time multi-agent consensus with communication delays, IET Control Theory and Applications, vol. 8, pp. 830-837, 2014.

[20] S. Li, Z. Xiang, H. Lin, and H. R. Karimi, "State estimation on positive Markovian jump systems with time-varying delay and uncertain transition probabilities," Information Sciences, vol. 369, pp. 251-266, 2016

[21] J. Liang, Z. Wang, and X. Liu, "State estimation for coupled uncertain stochastic networks with missing measurements and time-varying delays: the discrete- time case," IEEE Transactions on Neural Networks, vol. 20, pp. 781-793, 2009.

[22] S. Liu, G. Wei, Y. Song and Y. Liu, Extended Kalman filtering for stochastic nonlinear systems with randomly occurring cyber attacks, Neurocoomputing, Vol. 207, pp. 708-716, 2016.

[23] S. Liu, G. Wei, Y. Song and Y. Liu, Error-constrained reliable tracking control for discrete time-varying systems subject to quantization effects, Neurocoomputing, Vol. 174, Part B, pp. 897-905, 2016.

[24] X. Liu and T. Chen, "Synchronization of complex networks via aperiodically intermittent pinning control," IEEE Transactions on Automatic Control, vol. 60, no. 12, pp. 3316-3321, 2015.

[25] Y. Liu, Z. Wang, J. Liang, and X. Liu, "Synchronization and state estimation for discrete-time complex networks with distributed delays," IEEE Transactions on Systems, Man, and Cybernetics-Part B: Cybernetics, vol. 38, pp. 1314-1325, 2008.

[26] W. Lu and T. Chen, "Synchronization of coupled connected neural networks with delays," IEEE Trans. Circuits Syst. I, Reg. Papers, vol. 51, pp. 2491-2503, 2004.

[27] J. Lü and G. Chen, "A time-varying complex dynamical network model and its controlled synchronization criteria," IEEE Trans. Automatic Control, vol. 50, pp. 841-846, 2005.

[28] C. Lorand and P. H. Bauer, "On synchronization errors in networked feedback systems," IEEE Trans. Circuits Syst.I: Reg. Papers, vol. 53, pp. 2306-2317, 2006. 


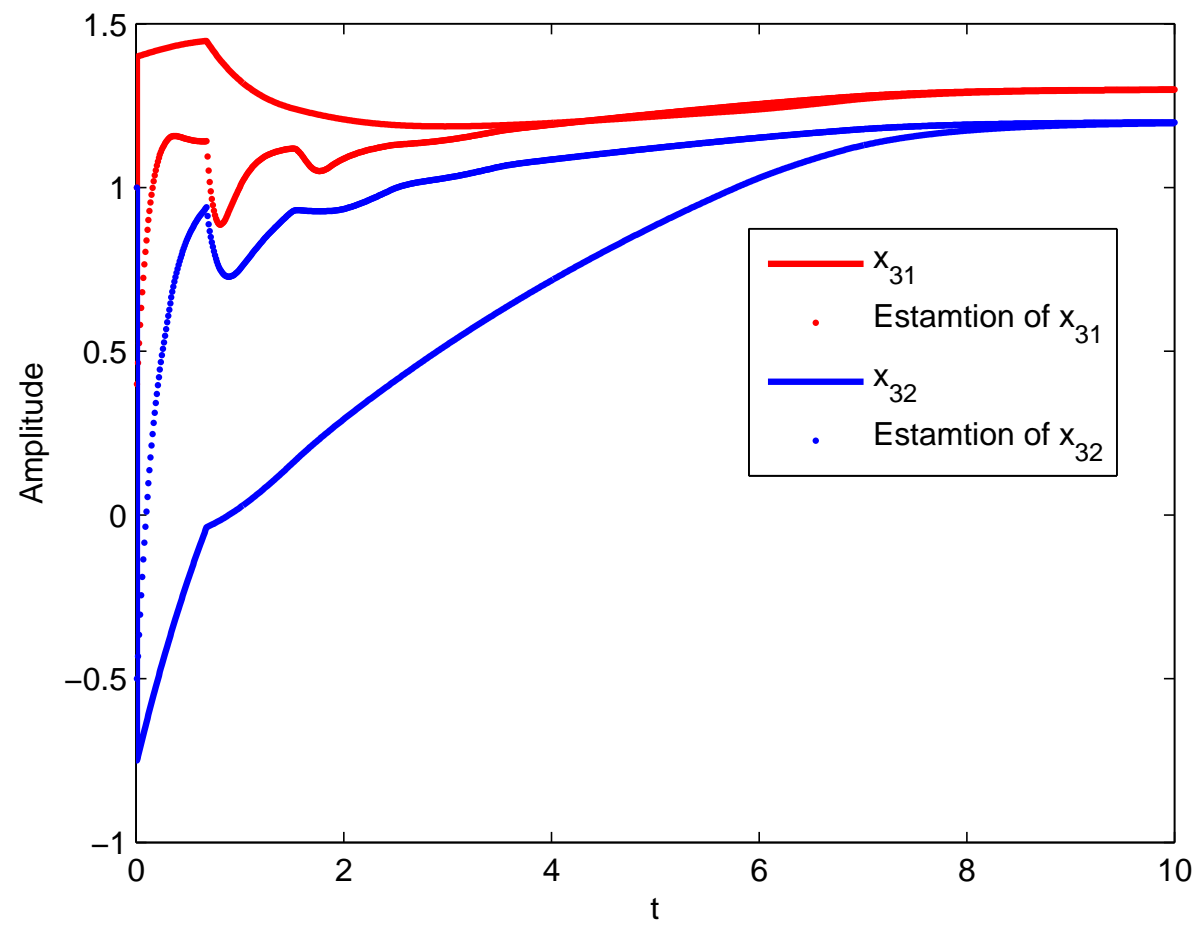

Fig. 3. State trajectories of $x_{3}$ and $\hat{x}_{3}$

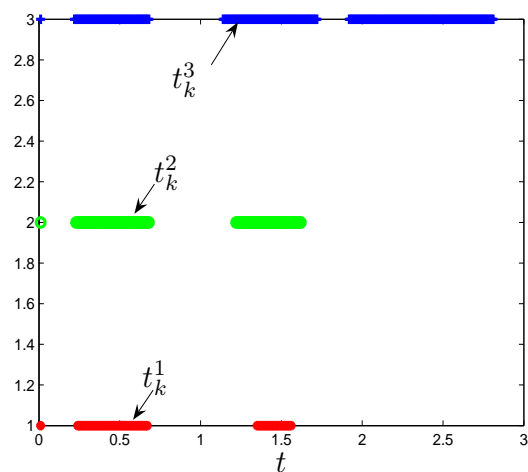

(a)

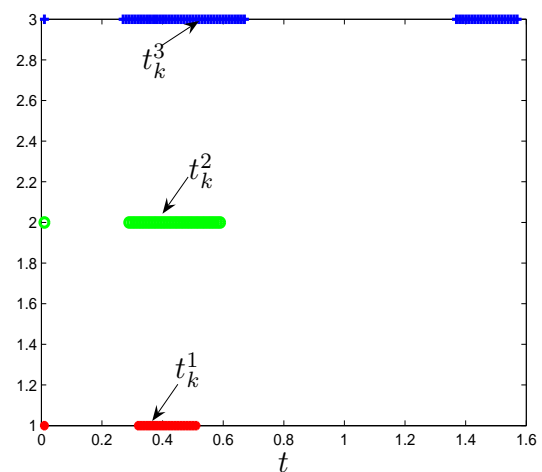

(b)

Fig. 4. Event-triggering instants with different $\delta_{i}$ : (a) triggering instants $t_{k}^{i}$ with $\delta_{i}=0.6$; (b) triggering instants $t_{k}^{i}$ with $\delta_{i}=0.8$.

[29] C. Peng and Q. Han, "A novel event-triggered transmission scheme and $\mathcal{L}_{2}$ control co-design for sampled-data control systems," IEEE Transactions on Automatic Control, vol. 58, pp. 2620-2626, 2013.

[30] X. Qing, H. R. Karimi, Y. Niu, and X. Wang, "Decentralized unscented Kalman filter based on a consensus algorithm for multi-area dynamic state estimation in power systems," International Journal of Electrical Power \& Energy Systems, vol. 65, pp. 26-33, 2014.

[31] R. Saber and R. Murray, "Consensus problems in networks of agents with switching topology and time-delays," IEEE Trans. Automat. Control, vol. 49, pp. 1520-1533, 2004.

[32] B. Shen, Z. Wang, D. Ding, and H. Shu, " $H_{\infty}$ state estimation for complex networks with uncertain inner coupling and incomplete measurements," IEEE Transactions on Neural Networks and Learning Systems, vol. 24, no. 12, pp. 2027-2037, 2013.

[33] Q. Song, Z. Zhao and Y. Liu, Impulsive effects on stability of discrete-time complex-valued neural networks with both discrete and distributed time-varying delays, Neurocomputing, Vol. 168, pp. 1044-1050, 2015.

[34] P. Tabuada, "Event-triggered real-time scheduling of stabilizing control tasks," IEEE Transactions on Automatic Control, vol. 52, pp. 1680-1685, 2007. 


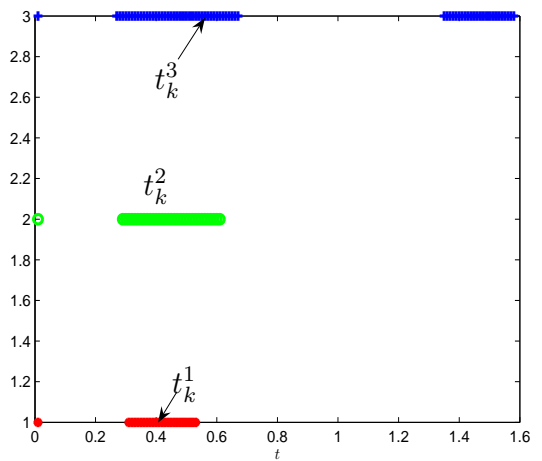

(a)

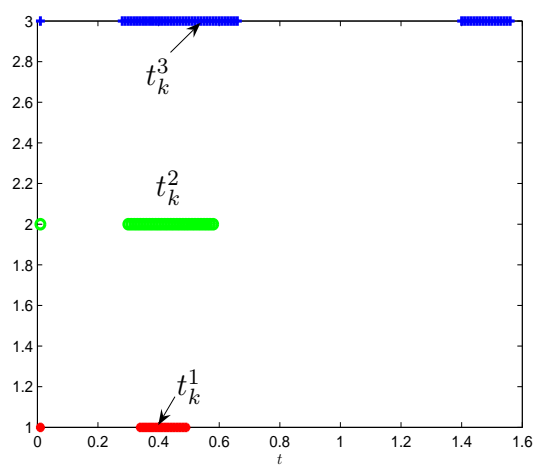

(b)

Fig. 5. Event-triggering instants with different $\beta$ : (a) triggering instants $t_{k}^{i}$ with $\beta=0$; (b) triggering instants $t_{k}^{i}$ with $\beta=0.2$.

[35] P. Tallapragada and N. Chopra, "Decentralized event-triggering for control of nonlinear systems," IEEE Transactions on Automatic Control, vol. 59, pp. 3312-3324, 2014.

[36] Y. Tang and W. K. Wong, "Distributed synchronization of coupled neural networks via randomly occurring control," IEEE Trans. Neural Networks and Learning Systems, vol. 24, pp. 435-447, 2013.

[37] L. Wang, G. Wei, and H. Shu, "State estimation for complex networks with randomly occurring coupling delays," Neurocomputing, vol. 122, pp. 513-520, 2013.

[38] D. Watts and S. Strogatz, "Collective dynamics of 'small-world' networks," Nature, vol. 393, pp. 440-442, 1998.

[39] X. Wang and M. Lemmon, "On event design in event-triggered feedback systems," Automatica, vol. 47, pp. 2319-2322, 2011.

[40] H. Yang, Z. Wang, H. Shu, F. E. Alsaadi and T. Hayat, Almost sure $H_{\infty}$ sliding mode control for nonlinear stochastic systems with Markovian switching and time-delays, Neurocomputing, Vol. 175, Part A, Jan. 2016, pp. 392-400.

[41] F. Yang, H. Dong, Z. Wang, W. Ren and F. E. Alsaadi, A new approach to non-fragile state estimation for continuous neural networks with time-delays, Neurocomputing, Vol. 197, pp. 205-211, Jul. 2016.

[42] Z. Yang and D. Xu, "Stability analysis and design of impulsive control systems with time delay," IEEE Trans. Automatic Control, vol. 52, pp. 1448-1454, 2007.

[43] D. Yue, E. Tian, and Q. Han, "A delay system method for designing event-triggered controllers of networked control systems," IEEE Transactions on Automatic Control, vol. 58, pp. 475-481, 2013.

[44] W. Zhang, Y. Tang, Q. Miao, and J. Fang, "Synchronization of stochastic dynamical networks under impulsive control with time delays," IEEE Trans. Neural Networks and Learning Systems, vol. 25, pp. 1758-1768, 2014.

[45] L. Zou, Z. Wang, H. Gao, and X. Liu, "Event-triggered state estimation for complex networks with mixed time delays via sampled data information: the continuous-time case," IEEE Transactions on Cybernetics, vol. 122, pp. 513-520, 2015. 